\title{
Imaging and quantifying the morphology of an organic-inorganic nanoparticle at the sub-nanometre level
}

\author{
Matti M. van Schooneveld ${ }^{1 \star}$, Alexandre Gloter², Odile Stephan², Luiz F. Zagonel², Rolf Koole ${ }^{3}$, \\ Andries Meijerink ${ }^{3}$, Willem J. M. Mulder ${ }^{4}$ and Frank M. F. de Groot ${ }^{1 \star}$
}

\begin{abstract}
The development of hybrid organic-inorganic nanoparticles is of interest for applications such as drug delivery, DNA and protein recognition, and medical diagnostics. However, the characterization of such nanoparticles remains a significant challenge due to the heterogeneous nature of these particles. Here, we report the direct visualization and quantification of the organic and inorganic components of a lipid-coated silica particle that contains a smaller semiconductor quantum dot. High-angle annular dark-field scanning transmission electron microscopy combined with electron energy loss spectroscopy was used to determine the thickness and chemical signature of molecular coating layers, the element atomic ratios, and the exact positions of different elements in single nanoparticles. Moreover, the lipid ratio and lipid phase segregation were also quantified.
\end{abstract}

$\mathrm{H}$ ybrid organic/inorganic nanoparticles based on polymers, inorganic materials and naturally occurring biomolecules offer a multitude of advantages for nanophotonics, catalysis and nanomedicine ${ }^{1-4}$. When used as multifunctional agents for biomedical applications, increased biocompatibility ${ }^{5}$ can be achieved, and the possibility of integrating different (contrast-generating) materials in the same unit allows their detection in a multimodal setting ${ }^{6,7}$. Examples of nanoconstructs that are simultaneously detectable by different modalities, such as fluorescent techniques, magnetic resonance imaging (MRI), positron emission tomography (PET) and/or X-ray computed tomography (CT) have been successfully applied in $v i v o^{8-10}$. Although the inorganic parts of hybrid nanoparticles can be readily characterized by conventional electron microscopic techniques, the exact structural arrangement of the organic molecular components remains largely unknown, and therefore the exact build-up of a hybrid particle remains unexplored $^{11}$. At best, studies on lipid-based particles have shown the direct visualization of lipid phase segregation on the nanometre scale for supported and unsupported giant unilamellar vesicles (GUVs) using atomic force microscopy ${ }^{12,13}$, and on the micrometre scale using coherent anti-stokes Raman scattering microscopy ${ }^{14}$ and fluorescent techniques ${ }^{15-17}$. These techniques are, however, only capable of visualizing the outer lipid layers of micrometresized particles.

Here, we present the simultaneous visualization and quantification of the sub-nanometre morphology of hybrid nanoparticles consisting of a quantum dot (QD)-containing silica particle coated by a dense paramagnetic and poly(ethylene glycol)-functionalized lipid monolayer. Previously, we developed this particle as an efficient and versatile contrast agent for fluorescence and MR imaging in vitro and in vivo ${ }^{5,18,19}$. The nanohybrid synthesis involves three coating steps as shown schematically in Fig. 1a (see Supplementary Information for synthesis details).
Core-shell-shell CdSe/CdS/Cd $\mathrm{Cd}_{0.5} \mathrm{ZnS}_{0.5} / \mathrm{ZnS}$ QDs are first incorporated in a silica shell that is subsequently made hydrophobic by covalent attachment of the octadecanol hydroxyl groups to the silica surface. The hydrophobic silica is then coated by a dense monolayer of equimolar amounts of paramagnetic gadolinium diethylene triamine pentaacetic acid di(stearylamide) ((Gd)-DTPA-DSA) and poly(ethylene glycol)-functionalized 1,2-distearoyl-sn-glycero-3phosphoethanolamine- $N$-[methoxy(poly(ethylene glycol))-2000] (PEG-DSPE) lipids (see Supplementary Fig. S1 for structure formulae of the lipids). High-angle annular dark-field (HAADF) electron microscopy images of a hydrophilic $\mathrm{QD} @ S i O_{2}$, a QD@SiO ${ }_{2} @$ octadecane and a QD@SiO $@$ @lipids particle are shown in Fig. $1 \mathrm{~b}-\mathrm{d}$, respectively. The HAADF images have a spatial resolution of $5 \AA$. The QDs used had an average diameter \pm s.d. of $7.0 \pm 0.5 \mathrm{~nm}$ and the $\mathrm{QD} @ \mathrm{SiO}_{2}$ particles had a mean size of $33.6 \pm 4.3 \mathrm{~nm}$ as determined by transmission electron microscopy (TEM; Supplementary Fig. S2). No difference was observed between the hydrophilic and hydrophobic QD@SiO particles. After coating with the lipids, many intense spots were observed around the particles; these had a mean spot diameter of $3.65 \pm 0.38 \mathrm{~nm}$.

Imaging of organic and inorganic parts of a nanoparticle The origin of the abovementioned spots was revealed using an electron energy loss spectroscopy (EELS) spectrum imaging approach ${ }^{20-22}$, which means that at every pixel of an image a full EELS spectrum was recorded. From this, element-specific chemical maps were constructed (a basic schematic of an EELS event is given in Supplementary Fig. S3) ${ }^{23}$. In combination with scanning transmission electron microscopy (STEM), EELS studies have proven to be advantageous in the study of biological systems ${ }^{24-27}$ and have also unveiled single atoms ${ }^{28-32}$. Figure 2 a displays a HAADF micrograph in which the intensity scale has been truncated to enhance the

\footnotetext{
'Inorganic Chemistry \& Catalysis, Debye Institute, Utrecht University, Sorbonnelaan 16, 3584 CA, The Netherlands, ${ }^{2}$ Laboratoire de Physique des Solides, Bâtiment 510, CNRS UMR 8502, Université Paris Sud XI, F 91405 Orsay, France, ${ }^{3}$ Condensed Matter \& Interfaces, Debye Institute, University Utrecht, PO Box 80000, 3508 TA Utrecht, The Netherlands, ${ }^{4}$ Translational and Molecular Imaging Institute, Mount Sinai School of Medicine, One Gustave L. Levy Place, New York, New York 10029, USA. *e-mail: M.M.vanSchooneveld@uu.nl; F.M.F.deGroot@uu.nl
} 

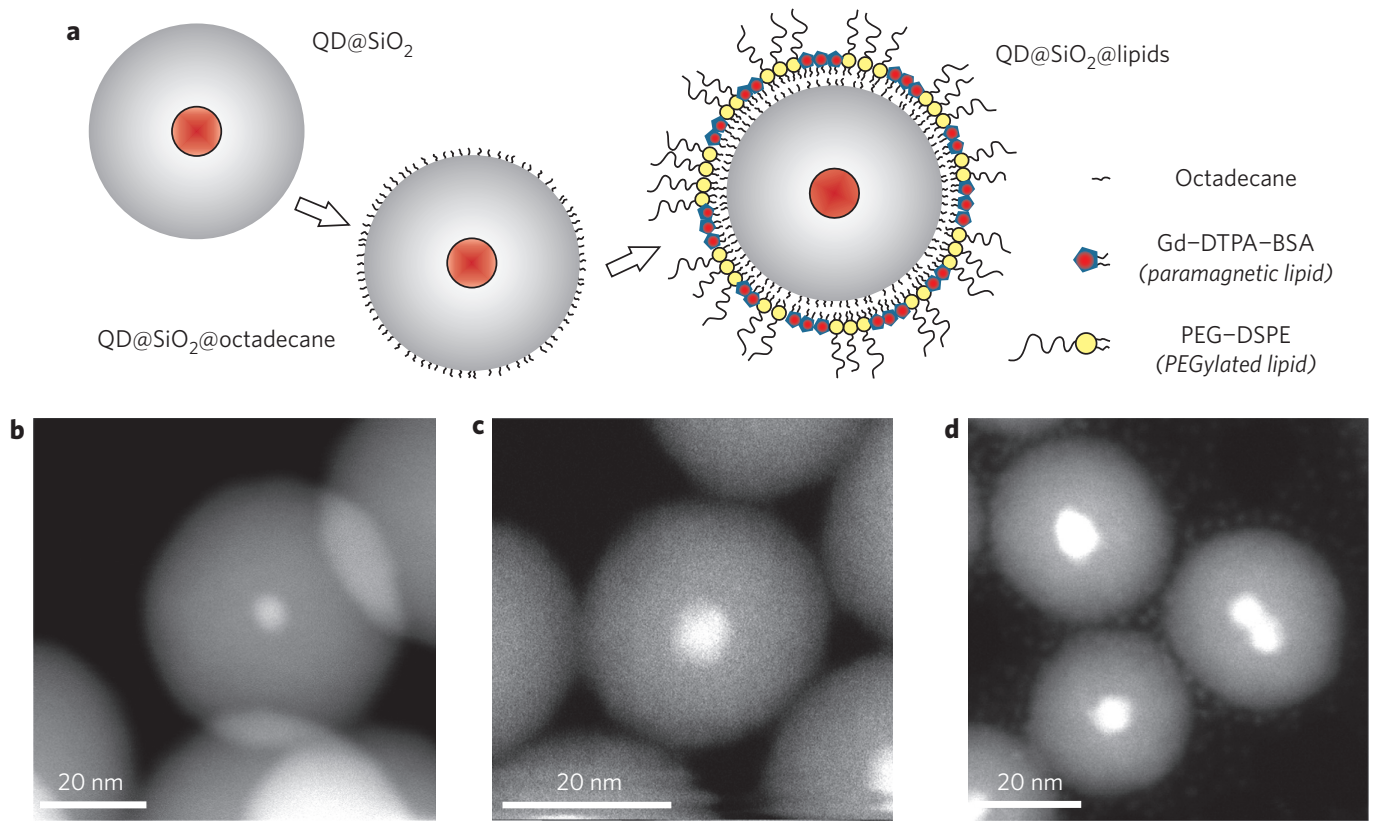

Figure 1 | Overview of the intermediate steps involved in the synthesis of lipid-coated, QD-containing silica nanoparticles. a, In the coating procedure, the QD-containing silica nanoparticles are first made hydrophobic by covalently attaching octadecane chains to the silica surface. Subsequently, paramagnetic Gd-DTPA-DSA and poly(ethylene glycol)-containing PEG-DSPE lipids are applied to coat the hydrophobic QD@SiO 2 nanoparticles with a monolayer of lipids. This nanoparticle platform serves as a bio-applicable, multimodal contrast agent for MRI and fluorescent techniques. b, HAADF electron micrograph of a bare, hydrophilic QD@SiO ${ }_{2}$ nanoparticle. The QD in the core of the silica nanoparticle is clearly visible. c, The octadecane layer could not be observed in the HAADF electron micrograph of hydrophobic QD@SiO ${ }_{2} @$ octadecane.d, Electron microscopy HAADF image of QD@SiO ${ }_{2} @$ lipids. Electron-intense white spots of gadolinium are observed around the nanoparticles with an average spot diameter of $3.65 \pm 0.38 \mathrm{~nm}$ (number of measurements $(n)=200 ; \pm$ indicates s.d.).

visibility of the spots around the $\mathrm{QD} @ S i O_{2} @$ lipids particles (which renders the QDs invisible). Figure $2 \mathrm{~b}$ shows a colour composite image of chemical maps of the area shown in Fig. 2a. The colours indicate electrons scattered by gadolinium (red), carbon (green) and silicon (blue) atoms. The chemical maps display electron intensities integrated over the $\mathrm{N}_{4,5}$ edge for gadolinium, the $\mathrm{K}$ edge for carbon and the $\mathrm{L}_{2,3}$ edge for silicon. Chemical map acquisition details can be found in the Methods. It follows that the electron intense spots around the lipid-coated QD@SiO ${ }_{2}$ nanoparticles were generated by gadolinium atoms, which were part of the GdDTPA-DSA lipids. Taking probe size convolution effects into account, which dominate over scattering delocalization effects ${ }^{33}$, we estimate the gadolinium spots, appearing as $3.65 \pm 0.38 \mathrm{~nm}$, to be $\sim 3.15 \mathrm{~nm}$. Using a surface area of $0.6 \mathrm{~nm}^{2}$ per lipid (refs 34,35 ) and approximating the gadolinium-rich spots to be circular, they were estimated to contain $\sim 13$ Gd-DTPA-DSA lipids each. This strongly suggests lipid-phase segregation in the lipid layer of the QD@SiO ${ }_{2} @$ lipids particles. Examination of other elements revealed a carbon signal at the nanoparticle surface, extending into the vacuum from the surfaces. Silicon atoms were clearly colocalized with the silica shell, while the presence of a dense core of a different material could be deduced from the decreased silicon signal at their centres. Figure $2 \mathrm{c}$ shows the obtained and matching EELS spectra of the cadmium $\mathrm{M}_{4,5}$, the silicon $\mathrm{L}_{2,3}$, the carbon $\mathrm{K}$ and the gadolinium $\mathrm{N}_{4,5}$ edges. The spectra were obtained summing over all the pixels of a chemical map. The insets show the corresponding chemical maps and reveal the presence of cadmium at the nanoparticle centre, originating from the cadmium-containing QDs. The silicon spectrum has its main $\mathrm{L}_{2,3}$ edge at $108.0 \mathrm{eV}$, which is a $7.5 \mathrm{eV}$ chemical shift compared to elemental silicon. This is due to the presence of silicon in its oxidized form silica $\left(\mathrm{SiO}_{2}\right)$. The carbon spectrum taken on the octadecane and lipid layer showed that the molecular bonds within the organic molecules were mostly damaged. Saturated carbon chains should not have a
$\mathrm{C}=\mathrm{C} \pi^{*}$ excitation at $285.5 \mathrm{eV}$, as observed here, which results from radiation damage ${ }^{36}$. However, additional spectral features were found indicative of the lipid layer such as the $\mathrm{C}-\mathrm{O} \sigma^{*}$ $(\sim 291.7 \mathrm{eV})$ and $\mathrm{C}=\mathrm{O} \sigma^{\star}(\sim 304.0 \mathrm{eV})$ excitations, which allow for the identification of the carbon as originating from the lipids.

To underpin the effects of electron beam damage on the lipid layer and the spatial distribution of the lipids in detail, measurements were performed using a liquid-nitrogen-cooled cryo-stage installed on the same STEM and with varying electron doses. Figure 3a shows the carbon K EELS spectra of the octadecane and lipid layers around the QD@SiO $@$ @lipids particles at $150 \mathrm{~K}$ as a function of electron dose. At the low dose of $2 e \AA^{-2}$, the carbon $\mathrm{K}$ ionization edge of the undamaged octadecane and lipid layer is shown. No peaks are observed between 285 and $288 \mathrm{eV}$, as expected for primary saturated lipid chains. The observed peaks at 289 and $292-293 \mathrm{eV}$ originate from $\mathrm{C}-\mathrm{C}$ and $\mathrm{C}-\mathrm{O} \sigma$ excitations within the intact lipids. At doses of 20 and $120 e \AA^{-2}$, a peak appears at $285.5 \mathrm{eV}$ and a shoulder at $288.5 \mathrm{eV}$. These excitations are ascribed to the formation of $\mathrm{C}=\mathrm{C}$ bonds that allow for $\pi^{*}$ transitions (carbon $1 s$ to $\pi^{*}{ }_{(\mathrm{C}=\mathrm{C})}$ at $285.5 \mathrm{eV}$ and $288.5 \mathrm{eV}$ ) due to the physical removal of hydrogen atoms by electron knock-on damage, particularly from the $\mathrm{CH}_{2}$ groups within the lipid saturated chains. When increasing the electron dose further, peaks related to the formation of $\mathrm{C}=\mathrm{O}$ bonds in the PEG-lipid chains occur (due to knock-on damage), first at $291 \mathrm{eV}$ and then at $288 \mathrm{eV}$, and are attributable to carbon $1 s$ to $\pi^{*}(\mathrm{C}=\mathrm{O})$ transitions. At doses at which the spectrum images and chemical maps in Figs 2, 4 and 5 were recorded $\left(2 \times 10^{5} e^{-2}\right)$, the $\mathrm{C}=\mathrm{O}$ molecular bonds still give spectral fine structure that allows the distinction between carbon resulting from the original lipids and amorphous carbon. The distinction between the two types of carbon based on their carbon K EELS spectra is also evident in spectrum images taken at lower electron doses (600 and $2 \times 10^{4} e \AA^{-2}$ ) and spatial resolution as shown in Supplementary Fig. S4. Although beam damage does thus degrade 

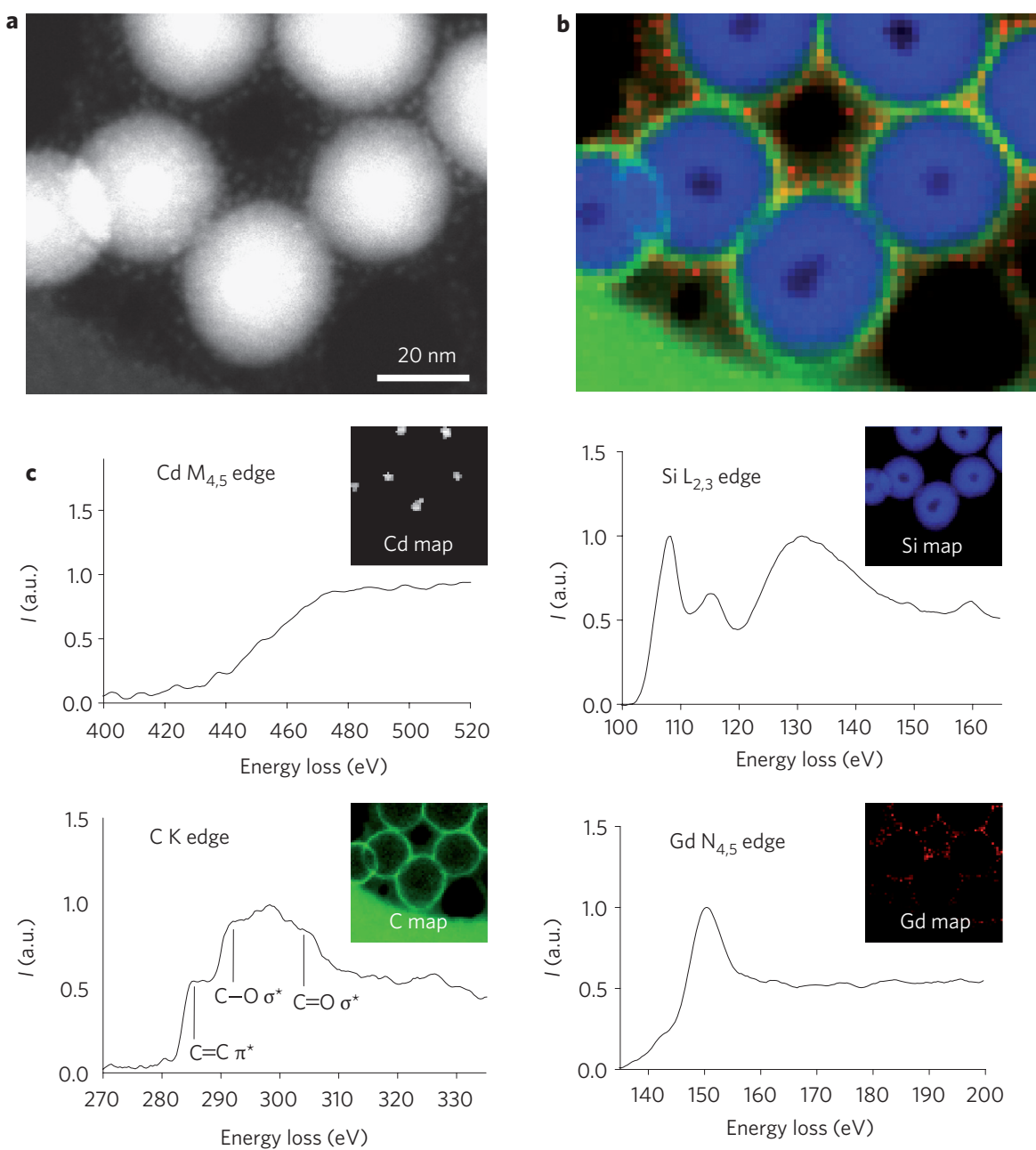

Figure 2 | Spatially resolved element analysis of the hybrid QD@SiO ${ }_{2} @$ lipids nanoparticles. a, HAADF image of the lipid-coated QD@SiO 2 nanoparticles. b. Composite colour map showing the location of different elements for the region in a. Red, blue and green indicate gadolinium, silicon and carbon atoms, respectively. The colour map is made by merging three chemical maps that only contain electron intensities due to inelastic scattering of the source electrons with the gadolinium $\mathrm{N}_{4,5}$, the silicon $\mathrm{L}_{2,3}$ and the carbon $\mathrm{K}$ core electrons. The green area in the bottom left corner represents carbon originating from the polymer film of the holey carbon electron microscopy grid. $\mathbf{c}$, EELS of the cadmium $\mathrm{M}_{4,5}$, the silicon $\mathrm{L}_{2,3}$, the carbon $\mathrm{K}$ and the gadolinium $\mathrm{N}_{4,5}$ edges. The spectra were obtained by averaging the spectra of the regions indicated in the chemical maps that are shown as insets. The coloured chemical maps show only electron intensities correlating to the abundance of the indicated specific element. The intensity (I) is given in arbitrary units (a.u.).

the lipid layer under the conditions needed to perform sub-nanometre STEM-EELS spectrum image analysis, it does not prevent the identification of whether carbon originates from the lipid layer. Importantly, Fig. $3 \mathrm{~b}$ shows a HAADF image obtained at $150 \mathrm{~K}$ and with an electron dose of $8 e \AA^{-2}$. Owing to the low applied dose, the electron intense spots in the lipid layer, which were shown to be gadolinium-rich in Fig. 2b,c, are not easily observed (left panel). However, when truncating the intensities of this low-temperature and low-dose HAADF image, the electron intense spots are clearly visible (right panel; the same HAADF image with four different intensity truncations can be found in Supplementary Fig. S5). These spots were also observed in Figs 1 and 2, recorded at high dose and without the cryo-stage, which implies that the gadolinium-rich domains are a real feature of the QD@SiO $2 @$ lipids nanoparticles. No spatial redistribution of the lipids within the lipid layer can be observed as a result of the high-intensity electron beam. It is therefore likely that the lipidlayer elemental composition is not significantly altered, and therefore an advanced and detailed characterization of the hybrid inorganic/organic nanoparticles is feasible with the STEM-EELS spectrum imaging approach.

\section{Analysing the nanoparticle build-up}

Figure $4 \mathrm{a}-\mathrm{c}$ presents colour composite maps of $\mathrm{QD} @ \mathrm{SiO}_{2}$, QD@SiO ${ }_{2} @$ octadecane and $\mathrm{QD}_{\mathrm{S}} \mathrm{SiO}_{2} @$ lipids particles. Analysis of the carbon signal during the different steps of the nanoparticle synthesis is particularly interesting. For the bare QD@SiO $\mathrm{O}_{2}$ nanoparticles in Fig. 4a almost no carbon was detected. Figure $4 \mathrm{~b}$ reveals a much stronger carbon signal, indicating that the octadecane coating was applied efficiently, whereas Fig. 4c shows a spatially extended carbon signal originating from the lipid coating, as well as the presence of gadolinium atoms. Chemical maps thus allow us to visualize the different coating steps involved in the preparation of these hybrid lipid-inorganic nanoparticles. Moreover, quantitative element analysis gives insight into the element spatial distribution and the particle dimensions. Figure $4 \mathrm{~d}-\mathrm{f}$ displays such distributions along the indicated lines in Fig. $4 a-c$. The element line profiles were quantified relative to the maximum amount of silicon found along the line (which was set to unity) and thus represent atomic ratios (see Methods for the quantification procedure). In Fig. 4d, only a weak and randomly distributed carbon signal is again observed for the uncoated particles. As well as the strong increase in the detected amount of carbon for the 

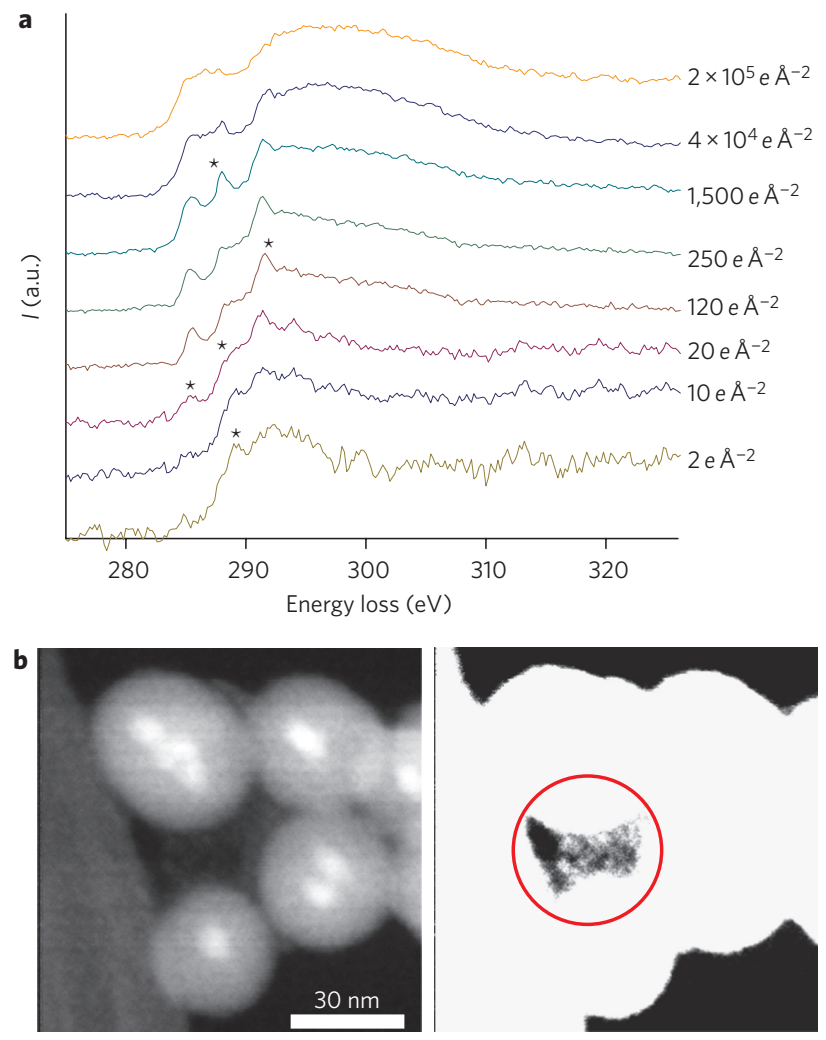

Figure 3 | Effect of the electron beam on the lipid layer of the hybrid inorganic/organic QD@SiO ${ }_{2} @$ lipids nanoparticles. a, Carbon K EELS spectra of the octadecane and lipid layer are shown as a function of applied electron dose. Although degradation of the lipid layer is observed with increasing dose, at the typical dose at which the spectrum images were acquired $\left(2 \times 10^{5}\right.$ e $\left.\AA^{-2}\right)$, carbon spectra can still be distinguished from amorphous carbon. The asterisks $\left(^{*}\right)$ indicate electron excitations that are discussed in the text. b, A HAADF electron micrograph of QD@SiO ${ }_{2} @$ lipids nanoparticles at $150 \mathrm{~K}$ and at a low electron dose of 8 e $\AA^{-2}$. Owing to this low dose and the large intensity range in the image, the gadolinium-rich domains are not easily observed (left panel), but they are revealed with a truncated intensity scale (right panel). The electron-intense and gadoliniumrich domains are thus observed at low temperature and dose, as was the case for the high-dose HAADF images of Figs 1d and 2a. The red circle indicates the region of interest.

QD@SiO ${ }_{2} @$ octadecane particle in Fig. 4e, we also observed an expected twofold increase $(1: 1.9)$ in the integrated carbon intensity between two silica particles compared to the free-lying side of the particle. This confirms that the detected carbon signal is quantitative and indeed originates from the octadecane layers of two particles. Furthermore, the mean distance between adjacent QD@SiO $@$ @octadecane particles in a set of HAADF images was measured to be $1.0 \pm 0.2 \mathrm{~nm}$ and the average carbon layer thickness $2.4 \pm 0.4 \mathrm{~nm}$. This observation, together with the measured doubling of the amount of carbon between two particles, leads to the conclusion that the octadecane chains had interpenetrated between the two particles. After applying the monolayer of lipids, we observe a strong increase in carbon signal for the resulting QD@SiO ${ }_{2} @$ lipids particles as shown in Fig. 4f. The gadolinium content was observed to peak just beyond the highest carbon signal. This result can be explained in terms of length differences between the Gd-DTPA-DSA and PEG-DSPE lipids. The gadolinium atoms are chelated in a DTPA complex at the end of two saturated hydrocarbon chains of the Gd-DTPA-DSA lipids. Until the point at which the shorter lipid ends (Gd-DTPA-DSA), a close- packed layer of hydrocarbon chains of both lipids is expected; moving further away from the silica surface reveals the presence of just one of the lipids, that is, the PEG-DSPE lipids. The gadolinium peak is therefore expected to be found directly after the maximum carbon density. Furthermore, cadmium and gadolinium were, as expected, less abundant than carbon and silicon. It should also be noted that more carbon and gadolinium were detected at the outer rims of the two-dimensional nanoparticle projections than in the centre of the particles (Fig. 4e,f). There are two main causes of this. First, more octadecane molecules and lipids are encountered by electrons travelling adjacent to the inorganic particle cores than by electrons travelling through them. Second, the attenuation of electron intensity through the electron-dense $\mathrm{QD} @ S i O_{2}$ part is greater than in organic parts of the nanohybrids. Figure $4 \mathrm{~g}$ presents histograms of the measured lipid layer thickness above the holes and above the polymer film of the holey carbon grids. In vacuum (above the holes), the average lipid layer thickness was $8.0 \pm 2.4 \mathrm{~nm}$, and on the carbon film a mean thickness of $19.4 \pm 3.8 \mathrm{~nm}$ was found. The lipid layer therefore tends to spread over the flat polymer film, as shown by the gadolinium traces observed around the silica particles in the inset of Fig. $4 \mathrm{~g}$ and in Supplementary Fig. S6, so only lipidcoated particles present in holes of the microscopy grids were studied here. Figure $4 \mathrm{~h}$ shows the carbon $\mathrm{K}$ spectra for the QD@SiO ${ }_{2}$, QD@SiO ${ }_{2} @$ octadecane and QD@SiO $\mathrm{S}_{2} @$ lipids particles, and a reference spectrum of amorphous carbon. For the bare QD@SiO ${ }_{2}$ nanoparticles the spectrum was of very weak intensity and little carbon was therefore present. Furthermore, the detected carbon did not result from carbon contamination because no $\pi^{\star}$ excitation of unsaturated $\mathrm{C}=\mathrm{C}$ bonds was observed at $285.5 \mathrm{eV}$ and the carbon fine structure was stable under the electron beam. The sharp peak at $288 \mathrm{eV}$ is characteristic of a $\mathrm{C}=\mathrm{O}\left(\pi^{*}\right)$ excitation and may result from some unreacted ethyl orthosilicate groups (-Si-O- $\mathrm{CH}_{2}-\mathrm{CH}_{3}$; the silica precursor) within the silica. The carbon spectrum acquired on the QD@SiO $\mathrm{O}_{2} @$ octadecane nanoparticles showed a strong $\sigma^{\star}$ contribution above $290 \mathrm{eV}$, revealing the presence of various $\mathrm{C}-\mathrm{C}$ and $\mathrm{C}-\mathrm{H}$ bonds, whereas the weak $\pi^{*}$ shoulder at $287 \mathrm{eV}$ results from the formation of unsaturated $\mathrm{C}=\mathrm{C}$ bonds in a hydrogenated environment (transition from carbon $1 s$ to $\left.\pi^{*}(\mathrm{C}=\mathrm{C})\right)^{37,38}$. As discussed previously, the lipid layer around the lipid-coated $\mathrm{QD} @ \mathrm{SiO}_{2}$ particles is significantly damaged by the electron beam, but some $\mathrm{C}-\mathrm{O} \sigma^{\star}$ and $\mathrm{C}=\mathrm{O}$ $\sigma^{\star}$ excitations indicative of the lipid layer are observed. Also the $\mathrm{C}=\mathrm{C} \pi^{*}$ excitation is measured at a higher energy than the well-defined $\mathrm{C}=\mathrm{C} \pi^{\star}$ excitation of amorphous carbon $(285.5 \mathrm{eV})$ due to the hydrogenated environment.

\section{Element ratio determination on the single-particle level}

The strength of EELS for quantifying the composition of hybrid nanoparticles composed of inorganic and naturally occurring molecules is fully illustrated in Fig. 5. In Fig. 5a, all elements in the QD@SiO ${ }_{2} @$ lipids are quantified over relevantly large areas. Again, the element molar ratios are relative to the amount of silicon, which was set to one. Integrating the signals under the quantified element maps in Fig. 5a gave molar ratios of 100/272/1.4/4.1 for silicon/carbon/cadmium/gadolinium in the hybrid nanoparticle. These ratios are in line with the 100/192/0.4/0.7 ratios estimated from a theoretical model (see Supplementary Information for model details). For the QD@SiO ${ }_{2}$ and QD@SiO $@$ @octadecane nanoparticles, the molar ratios for silicon/carbon/cadmium were found to be $100 / 8.7 / 1.7$ and 100/89/1.0, respectively (quantified maps not shown), which again approximate the theoretical estimated ratios of 100/74/0.4 in case of the QD@SiO $@ @$ octadecane particles. The experimentally observed 3.1-fold carbon increase after lipid coating comes close to the 2.6-fold increase that was predicted. Further analysis of the QD@SiO $\mathrm{S}_{2} @$ lipids revealed that the gadolinium signal was much higher at local spots between particles than 

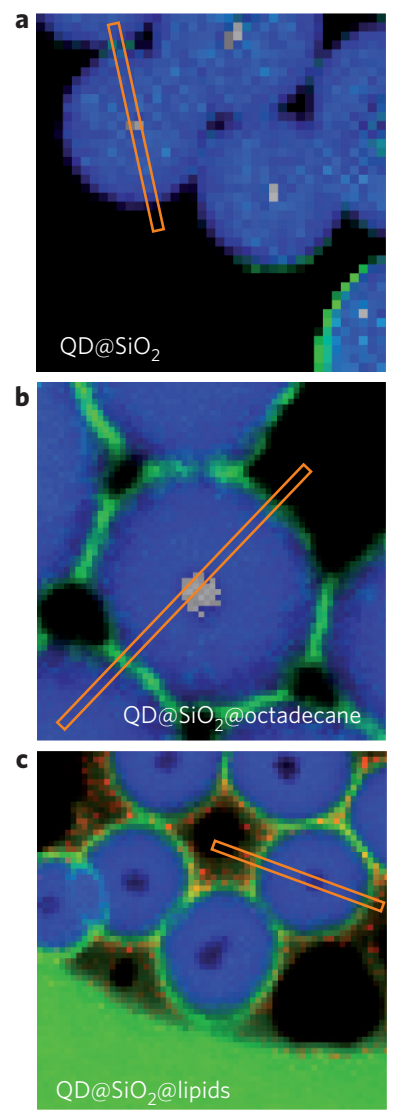
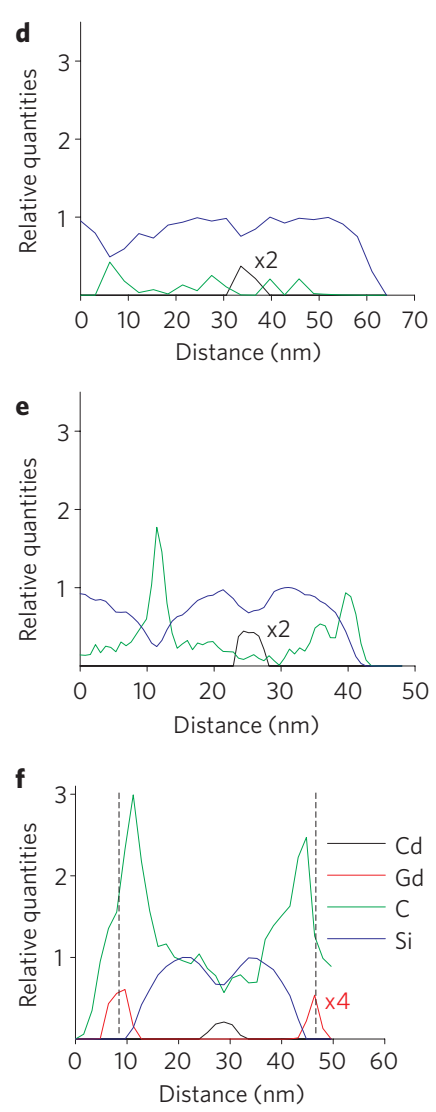
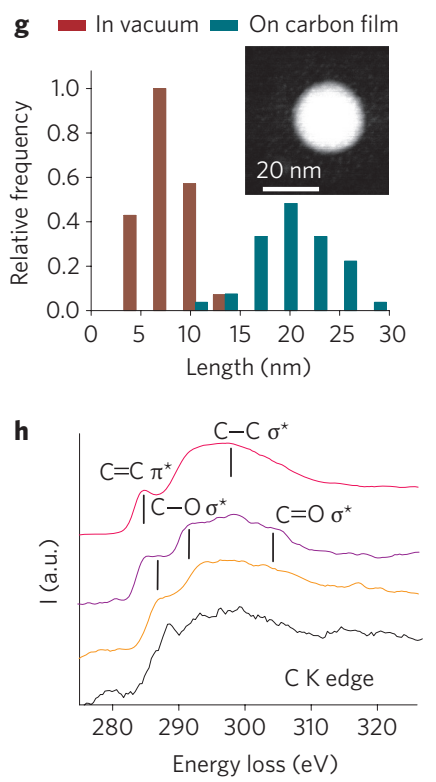

Figure 4 | Visualization, quantification and molecular characterization of the different steps involved in the synthesis of $\mathrm{QD}_{\mathrm{S}} @ \mathrm{SiO}_{2} @$ lipid nanoparticles. a, Composite colour image of three chemical maps of hydrophilic QD@SiO 2 nanoparticles. In panels a-c: grey pixels, cadmium; blue, silicon; green, carbon; red, gadolinium. b, Merged chemical maps of a QD@SiO ${ }_{2}$ nanoparticle after it has been coated with octadecane. c, Colour map of QD@SiO $@$ @lipid nanoparticles. Cadmium atoms are not shown. The green carbon area in the bottom-left corner originates from the polymer film of the holey carbon electron microscopy grid. $\mathbf{d}-\mathbf{f}$, Relative abundance of the different elements among the indicated rectangles in a-c for QD@SiO ${ }_{2}(\mathbf{d}), \mathrm{QD}_{\mathrm{S}} \mathrm{SiO}_{2} @$ octadecane (e) and $\mathrm{QD} @ \mathrm{SiO}_{2} @$ lipids (f) nanoparticles. The key to traces in $\mathbf{f}$ applies also to $\mathbf{d}$ and $\mathbf{e}$. The maximal detected amount of silicon is set to one and used to scale the relative abundance of the other elements. More carbon is observed after application of the octadecane (e) and the lipid coating (f), respectively. For the QD@SiO ${ }_{2} @ o c t a d e c a n e$ particles, the carbon signal is twice as low at a free-standing side of the nanoparticle than where it lies adjacent to another particle. For QD@SiO ${ }_{2} @$ lipid particles, the carbon signal extends furthest from the silica surface and the gadolinium atoms are observed just after the carbon peak in accordance with the model. Note that the gadolinium signal is multiplied by a factor of four in $\mathbf{f}$ to enhance the visibility of its position in the lipid layer (the cadmium signal is multiplied by two to enhance its visibility in $\mathbf{d}$ and $\mathbf{e}$ ). The dashed lines serve to visualize the position of the gadolinium atoms with respect to the maximum carbon density. g, Histograms of the lipid layer thickness of QD@SiO ${ }_{2} @$ lipids nanoparticles above the holes (brown) and on the

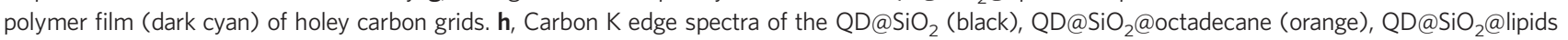
(violet) particles and a reference amorphous carbon spectrum (pink). The $\mathrm{QD} @ \mathrm{SiO}_{2} @$ lipids show the presence of $\mathrm{C}-\mathrm{O} \sigma^{\star}$ and $\mathrm{C}=\mathrm{O} \sigma^{\star}$ excitations and confirm the presence of the lipids.

at the free sides of particles. This result suggests that multiple lipid layers were confined in the small spaces between adjacent silica spheres, which is an important indication that single silica spheres, and not multiple spheres, are enveloped in a lipid layer (further evidence of this can be found in HAADF images in Supplementary Fig. S7). In Fig. 5b,c, STEM-HAADF was used to estimate the Gd-DTPA-DSA:PEG-DSPE lipid ratio. Figure 5b shows a HAADF image of a lipid layer between three $\mathrm{QD} @ \mathrm{SiO}_{2}$ particles. The colour-coded rectangle indicates a region where the lipid layer was relatively flat. A histogram of pixel intensities in this region can be found in Fig. 5c. The histogram shows two main contributions, one that is assumed to be a result of the low electron-intense PEG-lipids only and one that is ascribed to the high electron-intense Gd-lipids. By choosing a cut-off value between these peaks, PEG-lipid $(<1,500$ counts) and Gd-lipid $(>1,500$ counts) map were constructed, as shown in Fig. 5b. After integrating the pixel histogram below and above this value, as visualized in Fig. 5c, the molar Gd-DTPA-DSA:PEG-DSPE lipid ratio was estimated to be 0.34:0.66. This estimation is thus lower than the 0.5:0.5 molar ratio applied in the synthesis, which strongly suggests that the actual lipid ratio in the lipid layer around the $\mathrm{QD} @ \mathrm{SiO}_{2}$ particles does not only depend on the applied lipid ratio.

\section{Conclusions}

These results demonstrate that STEM-HAADF measurements, in combination with EELS, are capable of the simultaneous visualization of both the inorganic and organic parts of a hybrid inorganic/ organic nanoparticle with sub-nanometre resolution. The reported experiments give a deep qualitative insight into the morphology of the hybrid nanoparticles in their final form, as well as during the different steps of their synthesis. It thus reveals detailed information on the nanoparticle build-up or architecture, which is otherwise inaccessible. In addition, STEM-EELS allows for quantification of such nanohybrids on the single-particle level. The rich quantitative information that is obtained in this study is threefold. First, the molecular signature, relative amount and spatial extension of the carbon-containing layers were determined. Second, the Gd-lipid position and the number of gadolinium atoms in lipid patches at 
a
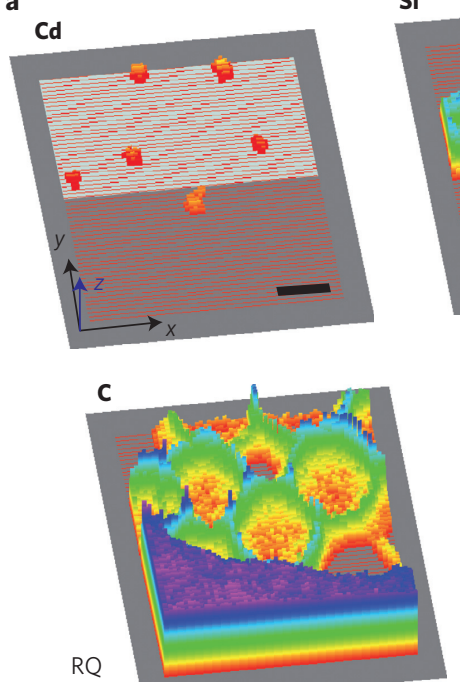

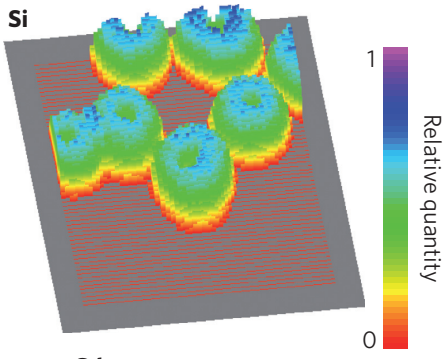

Gd

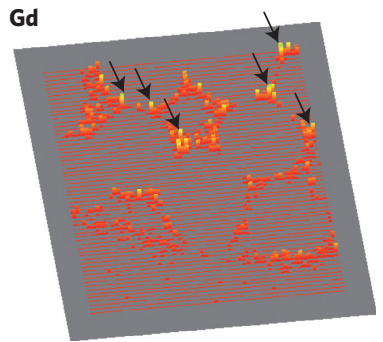

b

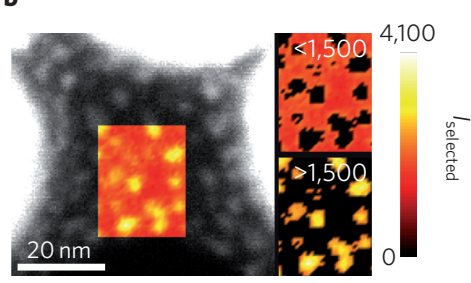

c

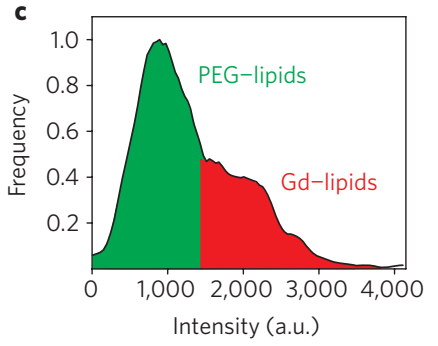

Figure 5 | Full-field, relative quantification of the different elements in the hybrid lipid-coated QD@SiO ${ }_{2}$ nanoparticles and of the lipid ratio in the lipid layer. a, Quantitative element maps of the QD@SiO ${ }_{2} @$ lipid nanoparticles indicating the location ( $x$ - and $y$-axes) and relative amounts ( $z$-axis) of cadmium, silicon, carbon and gadolinium atoms. The relative quantity (RQ) of carbon atoms was downscaled by a factor of four to enhance the visibility of the quantitative cadmium and gadolinium signals. The scale bar in the cadmium panel indicates $20 \mathrm{~nm}$ and applies to all panels. The light-shaded area in the cadmium panel indicates the region over which the element molar ratios were determined. The arrows in the gadolinium map indicate very high gadolinium

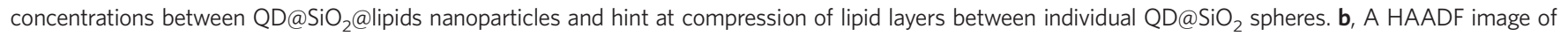
a lipid layer between three QD@SiO ${ }_{2}$ spheres. The colour-coded region indicates a region were the lipid layer is relatively flat. This region is used to estimate the Gd-DTPA-DSA and PEG-DSPE lipid molar ratio. c, Histogram of the pixel intensities in the colour-coded region of $\mathbf{b}$. Two contributions are predominantly observed. Low-intensity pixels belong to the PEG-DSPE lipids (green area) and high-intensity pixels are ascribed to the electron-intense Gd-DTPA-DSA lipids (red area). Selecting a cut-off value between the two contributions in the histogram enables lipid-specific maps to be made $(<1,500=$ PEG-lipids; $>1,500=$ Gd-lipids) as shown in $\mathbf{b}$ and allows an estimate of the Gd-lipid:PEG-lipid ratio of 0.34:0.66.

the surface of the nanoparticles were revealed. Moreover, in the final experiments the relative atomic silicon/carbon/cadmium/gadolinium ratios and the lipid ratios in the lipid layer of the hybrid nanoparticles were estimated.

Hybrid bio-nanoconstructs, such as the one presented here, are becoming increasingly important in many scientific disciplines, including nanomedicine. The chemical identification and quantification of such materials with sub-nanometre resolution is a welcome addition to the nanohybrid characterization toolbox. For the development of, for example, nanoparticulate contrast agents, the full characterization of particle composition will be crucial in establishing successful applications. Advanced developments in STEM-EELS, such as single atom detection using low accelerating voltages $^{32}$, sub-ångstrom resolution ${ }^{39,40}$, chemical bond mapping ${ }^{41}$, tomographic ${ }^{42}$ and femtosecond-resolved ${ }^{43}$ imaging may revolutionize the way we regard hybrid nanoparticles in days to come. Overall, we envision that this characterization tool can lead to an important understanding of a wide variety of complex hybrid organic/inorganic nanostructures.

\section{Methods}

STEM. The VG-HB501 STEM was equipped with a tungsten cold field-emission gun working at $100 \mathrm{keV}$. The beam convergence half angle was set to $7.5 \mathrm{mrad}$. This corresponds to the formation of the smallest possible electron probe ( $5 \AA$ in diameter) at the sample surface with a current of $\sim 0.1 \mathrm{nA}$. The half angle of collection was limited to $24 \mathrm{mrad}$ for chemical mapping and to $12 \mathrm{mrad}$ for carbon $\mathrm{K}$ fine structure analysis, where higher energy resolution is required. The postcolumn Gatan 666 parallel spectrometer ${ }^{44}$ was operated with a dispersion of 0.5 and $0.2 \mathrm{eV}$ per channel for chemical and carbon K-edge analysis. EELS spectra were recorded with a two-dimensional back-illuminated charge-coupled device camera $(1,340 \times 100$ pixels $)$ with a low read-out noise of three counts r.m.s. and a negligible dark count noise. The initial energy resolution of the electron beam was $\sim 0.3 \mathrm{eV}$. Experiments were carried out at room temperature if not stated otherwise.

EELS acquisition and processing. The data were acquired in the so-called spectrum imaging mode. The sub-nanometre probe was rastered in two-dimensions over the sample, and a whole EELS spectrum in the core-loss region $(70-570 \mathrm{eV})$ was acquired at each position of the probe. The dwell time was set between 50 and $300 \mathrm{~ms}$ per spectrum and a charge-coupled device binning of $1 \times 100$ was used. The binning conditions were chosen as a compromise between a substantial signal-tonoise ratio and a reasonable acquisition time per spectrum image (with the present conditions, the acquisition time was a few minutes for $32 \times 32$ two-dimensional spectrum images) to avoid major sample drift during the scan of the focused electron beam and to reduce radiation damage or contamination effects. For fine structure analysis of the carbon K-edge, the samples were maintained at $150 \mathrm{~K}$ by liquid-nitrogen cooling. The samples were not exposed to electrons before image or EELS acquisition, which was made possible by a fast blanking system before the sample. Electron doses ranging from 2 to $2 \times 10^{5} \mathrm{e} \mathrm{A}^{-2}$ were applied in the acquisition of several carbon $1 s$ EELS spectra. Otherwise, typical doses of respectively $5 \times 10^{2}$ and $2 \times 10^{5} e^{-2}$ were used to record the presented HAADF and spectrum images. Post-acquisition processing of the acquired data was carried out using DigitalMicrograph software. Chemical maps were made by integrating electron intensities of ionization edges, while correcting for the material-unspecific inelastic scattering background. The spectral images were normally calibrated to the $\mathrm{SiO}_{2} \mathrm{~L}_{2,3}$ edge at $108.0 \mathrm{eV}$ and the carbon signal at $285 \mathrm{eV}$. A typical energy range $(\Delta E)$ of $\sim 15 \mathrm{eV}$ was used from between -2 and $+13 \mathrm{eV}$ with respect to the determined edges. These edges were determined to be $108.0,140.0$ and $285.0 \mathrm{eV}$ for silicon, gadolinium and carbon, respectively. For the weak cadmium $\mathrm{M}_{4,5}$ edge, $\Delta E \approx 90 \mathrm{eV}$, starting at $405 \mathrm{eV}$. Electron energy spectra were obtained by performing a threshold operation on a chemical map and summing over all remaining pixels of the chemical map before being corrected for spectra taken in vacuum. Quantification of line profiles and full spectral images was carried out by correcting for the edge-specific partial inelastic scattering cross-sections, which were determined using a Hartree-Slater model included in the DigitalMicrograph software ${ }^{45}$. Using this, the number of atoms of a specific element relative to another element may be determined using equation (1) (ref. 33):

$$
\frac{N^{\mathrm{A}}}{N^{\mathrm{B}}}=\frac{I_{\mathrm{CL}}^{\mathrm{A}}(\beta, \Delta)}{I_{\mathrm{CL}}^{\mathrm{B}}(\beta, \Delta)} * \frac{\sigma_{\mathrm{CL}}^{\mathrm{B}}(\beta, \Delta)}{\sigma_{\mathrm{CL}}^{\mathrm{A}}(\beta, \Delta)}
$$

where $N^{\mathrm{A}}$ indicates $N$ atoms of element A per unit area. $I_{\mathrm{CL}}^{\mathrm{A}}(\beta, \Delta)$ is the integrated intensity of a core-loss (CL) event from the energy up to energy $\Delta$, for electrons scattered under an angle 0 to $\beta$ (the EELS spectrometer collection semiangle) and $\sigma_{\mathrm{CL}}^{\mathrm{A}}(\beta, \Delta)$ is the partial inelastic scattering cross-section dependent on $\beta$ and $\Delta$. 
Received 15 March 2010; accepted 26 April 2010; published online 6 June 2010

\section{References}

1. Caruso, F. Nanoengineering of particle surfaces. Adv. Mater. 13, 11-22 (2001).

2. Chowdhury, E. H. \& Akaike, T. Bio-functional inorganic materials: an attractive branch of gene-based nano-medicine delivery for 21 st century. Curr. Gene Ther. 5, 669-676 (2005).

3. Katz, E. \& Willner, I. Integrated nanoparticle-biomolecule hybrid systems: synthesis, properties and applications. Angew. Chem. Int. Ed. 43, 6042-6108 (2004).

4. You, C.-C., Chompoosor, A. \& Rotello, V. M. The biomacromoleculenanoparticle interface. Nano Today 2, 34-43 (2007).

5. van Schooneveld, M. M. et al. Improved biocompatibility and pharmacokinetics of silica nanoparticles by means of a lipid coating: a multimodality investigation. Nano Lett. 8, 2517-2525 (2008).

6. Rosi, N. L. \& Mirkin, C. A. Nanostructures in biodiagnostics. Chem. Rev. 105, 1547-1562 (2005).

7. Koole, R. et al. Magnetic quantum dots for multimodal imaging. Wiley Interdiscip. Rev. Nanomed. Nanobiotechnol. 1, 475-491 (2009).

8. Bridot, J.-L. et al. Hybrid gadolinium oxide nanoparticles: multimodal contrast agents for in vivo imaging. J. Am. Chem. Soc. 129, 5076-5084 (2007).

9. Lu, C. W. et al. Bifunctional magnetic silica nanoparticles for highly efficient human stem cell labeling. Nano Lett. 7, 149-154 (2007).

10. Jaffer, F. A., Libby, P. \& Weissleder, R. Optical and multimodality molecular imaging: insights into atherosclerosis. Arterioscler. Thromb. Vasc. Biol. 29, 1017-1024 (2009).

11. Richman, E. K. \& Hutchison, J. E. The nanomaterial characterization bottleneck. ACS Nano 3, 2441-2446 (2009).

12. Tokumasu, F., Jin, A. J., Feigenson, G. W. \& Dvorak, J. A. Nanoscopic lipid domain dynamics revealed by atomic force microscopy. Biophys. J. 84, 2609-2618 (2003).

13. Rinia, H. A. \& de Kruijff, B. Imaging domains in model membranes with atomic force microscopy. FEBS Lett. 504, 194-199 (2001).

14. Potma, E. O. \& Sunney Xie, X. Direct visualization of lipid phase segregation in single lipid bilayers with coherent anti-stokes Raman scattering microscopy. ChemPhysChem 6, 77-79 (2005).

15. Ariola, F. S., Mudaliar, D. J., Walvick, R. P. \& Heikal, A. A. Dynamics imaging of lipid phases and lipid-marker interactions in model biomembranes. Phys. Chem. Chem. Phys. 8, 4517-4529 (2006).

16. Dietrich, C. et al. Lipid rafts reconstituted in model membranes. Biophys. J. 80, 1417-1428 (2001).

17. Plasencia, I., Norlen, L. \& Bagatolli, L. A. Direct visualization of lipid domains in human skin stratum corneum's lipid membranes: effect of $\mathrm{pH}$ and temperature. Biophys. J. 93, 3142-3155 (2007).

18. Mulder, W. J. M. et al. Quantum dots with a paramagnetic coating as a bimodal molecular imaging probe. Nano Lett. 6, 1-6 (2006).

19. Koole, R. et al. Paramagnetic lipid-coated silica nanoparticles with a fluorescent quantum dot core: a new contrast agent platform for multimodality imaging. Bioconjug. Chem. 19, 2471-2479 (2008).

20. Jeanguillaume, C. \& Colliex, C. Spectrum-image: the next step in EELS digital acquisition and processing. Ultramicroscopy 28, 252-257 (1989).

21. Teunissen, W. et al. The structure of carbon encapsulated NiFe nanoparticles. J. Catal. 204, 169-174 (2001).

22. Catala, L. et al. Core-multishell magnetic coordination nanoparticles: toward multifunctionality on the nanoscale. Angew. Chem. Int. Ed. 48, 183-187 (2009).

23. Thomas, J. M., Williams, B. G. \& Sparrow, T. G. Electron-energy-loss spectroscopy and the study of solids. Acc. Chem. Res. 18, 324-330 (1985).

24. Leapman, R. D. \& Ornberg, R. L. Quantitative electron energy loss spectroscopy in biology. Ultramicroscopy 24, 251-268 (1988).

25. Egerton, R. F. Quantitative analysis of electron-energy-loss spectra. Ultramicroscopy 28, 215-225 (1989).

26. Engel, A. \& Colliex, C. Application of scanning transmission electron microscopy to the study of biological structure. Curr. Opin. Biotechnol. 4, 403-411 (1993).
27. Sousa, A. A. et al. Determining molecular mass distributions and compositions of functionalized dendrimer nanoparticles. Nanomedicine 4, 391-399 (2009).

28. Suenaga, K. et al. Element-selective single atom imaging. Science $\mathbf{2 9 0}$ 2280-2282 (2000)

29. Batson, P. E., Dellby, N. \& Krivanek, O. L. Sub-ångstrom resolution using aberration corrected electron optics. Nature 418, 617-620 (2002).

30. Spence, J. C. H. Absorption spectroscopy with sub-ångstrom beams: ELS in STEM. Rep. Prog. Phys. 69, 725-258 (2006).

31. Bosman, M. et al. Two-dimensional mapping of chemical information at atomic resolution. Phys. Rev. Lett. 99, 086102 (2007).

32. Suenaga, K. et al. Visualizing and identifying single atoms using electron energyloss spectroscopy with low accelerating voltage. Nature Chem. 1, 415-418 (2009)

33. Egerton, R. F. Electron energy-loss spectroscopy in the TEM. Rep. Prog. Phys. 72, 016502 (2009)

34. Petrache, H. I., Dodd, S. W. \& Brown, M. F. Area per lipid and acyl length distributions in fluid phosphatidylcholines determined by ${ }^{2} \mathrm{H}$ NMR spectroscopy. Biophys. J. 79, 3172-3192 (2000).

35. Schmitt, L., Dietrich, C. \& Tampe, R. Synthesis and characterization of chelatorlipids for reversible immobilization of engineered proteins at self-assembled lipid interfaces. J. Am. Chem. Soc. 116, 8485-8491 (2002).

36. Egerton, R. F., Li, P. \& Malac, M. Radiation damage in the TEM and SEM. Micron 35, 399-409 (2004).

37. Urquhart, S. G. et al. Inner-shell excitation spectroscopy of polymer and monomer isomers of dimethyl phthalate. J. Phys. Chem. B 101, 2267-2276 (1997)

38. Hitchcock, A. P. Bibliography of atomic and molecular inner-shell excitation studies. J. Electron Spectros. Relat. Phenomena 67, 1-132 (1994).

39. Krivanek, O. L. et al. An electron microscope for the aberration-corrected era. Ultramicroscopy 108, 179-195 (2008).

40. Muller, D. A. et al. Atomic-scale chemical imaging of composition and bonding by aberration-corrected microscopy. Science 319, 1073-1076 (2008)

41. Arenal, R. et al. Extending the analysis of EELS spectrum-imaging data, from elemental to bond mapping in complex nanostructures. Ultramicroscopy 109, 32-38 (2008).

42. Hohmann-Marriott, M. F. et al. Nanoscale 3D cellular imaging by axial scanning transmission electron tomography. Nature Methods 6, 729-731 (2009).

43. Carbone, F., Kwon, O.-H. \& Zewail, A. H. Dynamics of chemical bonding mapped by energy-resolved $4 \mathrm{D}$ electron microscopy. Science $\mathbf{3 2 5}$, 181-184 (2009).

44. Egerton, R. F., Yang, Y. Y. \& Cheng, S. C. Characterization and use of the Gatan 666 parallel-recording electron energy-loss spectrometer. Ultramicroscopy 48, 239-250 (1993).

45. Rez, P. Cross-sections for energy loss spectrometry. Ultramicroscopy $\mathbf{9}$, 283-287 (1982)

\section{Acknowledgements}

The authors would like to thank C. Morin, I. Swart, A. Juhin, E. de Smit and H. van Hattum for useful discussions. M. Kociak and M. Tencé are gratefully acknowledged for their help in designing the liquid-nitrogen STEM cooling stage. This work was financially supported by the I3 European project ESTEEM (no. 026019) and a VICI grant (F.M.F.d.G.) of the Netherlands Organization for Scientific Research (NWO-CW).

\section{Author contributions}

M.M.v.S. designed the experiment with help from F.M.F.d.G. M.M.v.S. synthesized the hybrid nanoparticles, processed the data and wrote the manuscript. A.G., O.S. and L.F.Z. performed the STEM-HAADF and EELS measurements, together with M.M.v.S. W.J.M.M., R.K., M.M.v.S. and A.M. played a major role in the design and development of the hybrid nanoparticles. All authors discussed the results and commented on the manuscript.

\section{Additional information}

The authors declare no competing financial interests. Supplementary information accompanies this paper at www.nature.com/naturenanotechnology. Reprints and permission information is available online at http://npg.nature.com/reprintsandpermissions/. Correspondence and requests for materials should be addressed to M.M.v.S. and F.M.F.d.G. 